\title{
Clinical and Cognitive Phenotype of Mild Cognitive Impairment Evolving to Dementia with Lewy Bodies
}

\author{
Annachiara Cagnin ${ }^{a}$ b Cinzia Bussè ${ }^{a}$ Simona Gardinic, d Nela Jelcic ${ }^{a, b}$ \\ Caterina Guzzo c, d Francesca Gnoato ${ }^{a}$ Micaela Mitolo ${ }^{b}$ Mario Ermani ${ }^{a}$ \\ Paolo Caffarrac, d \\ a Department of Neurosciences, University of Padova, Padova, ${ }^{b}$ IRCCS San Camillo Research \\ Hospital, Venice, and ${ }^{\mathrm{C}}$ Department of Neuroscience, University of Parma, and ${ }^{\mathrm{d}}$ Center for \\ Cognitive Disorders, AUSL of Parma, Parma, Italy
}

\author{
Key Words \\ Dementia with Lewy bodies - Visuoconstructional abilities - Mini Mental State Examination . \\ Mild cognitive impairment
}

\section{Abstract}

Objective: The aim of this study was to determine which characteristics could better distinguish dementia with Lewy bodies (DLB) from Alzheimer's disease (AD) at the mild cognitive impairment (MCI) stage, with particular emphasis on visual space and object perception abilities. Methods: Fifty-three patients with mild cognitive deficits that were eventually diagnosed with probable DLB (MCI-DLB: $n=25$ ) and AD (MCI-AD: $n=28$ ) at a 3-year follow-up were retrospectively studied. At the first visit, the patients underwent cognitive assessment including the Qualitative Scoring Mini Mental State Examination Pentagon Test and the Visual Object and Space Perception Battery. The Neuropsychiatric Inventory Questionnaire, Unified Parkinson's Disease Rating Scale (UPDRS) and questionnaires for cognitive fluctuations and sleep disorders were also administered. Results: The best clinical predictor of DLB was the presence of soft extrapyramidal signs (mean UPDRS score: $4.04 \pm 5.9$ ) detected in $72 \%$ of patients, followed by REM sleep behavior disorder (60\%) and fluctuations (60\%). Wrong performances in the pentagon's number of angles were obtained in 44\% of DLB and $3.7 \%$ of AD patients and correlated with speed of visual attention. Executive functions, visual attention and visuospatial abilities were worse in DLB, while verbal episodic memory impairment was greater in AD. Deficits in the visual-perceptual domain were present in both MCI-DLB and AD. Conclusions: Poor performance in the pentagon's number of angles is specific of DLB and correlates with speed of visual attention. The dorsal visual stream seems specifically more 
Cagnin et al.: Clinical and Cognitive Phenotype of Mild Cognitive Impairment Evolving to Dementia with Lewy Bodies

impaired in MCI-DLB with respect to the ventral visual stream, the latter being involved in both DLB and AD. These cognitive features, associated with subtle extrapyramidal signs, should alert clinicians to a diagnostic hypothesis of DLB.

(c) 2015 The Author(s)

Published by S. Karger AG, Basel

\section{Introduction}

Although a distinct cognitive profile in patients with dementia with Lewy bodies (DLB) and dementia due to Alzheimer's disease (AD) has been extensively demonstrated (worse memory domain in AD and attentional/visuospatial domain in DLB), only few studies have addressed the same topic in the prodromal stage of these diseases using extensive and comprehensive cognitive tools [1-3].

The pentagon copying of the Mini Mental State Examination (MMSE) was investigated for the first time in DLB and AD patients [4], then updated with an analytic approach and validated in a neuropathologically proven DLB cohort $[5,6]$. In the study by Ala et al. [4], an unacceptable pentagon copy reached an $88 \%$ sensitivity and a $58 \%$ specificity in discriminating the two conditions. It was also recognized that this feature could be better captured in the early stages of the diseases, and it was suggested that it may be independent of other wellrecognized diagnostic characteristics of DLB. On this line, a previous study from our group demonstrated that a reduced number of angles of the MMSE pentagon copy could be a marker of prodromal DLB with a specificity of $91 \%$ in the discrimination with prodromal AD, suggesting that visuoconstructional impairment may serve as an early cognitive marker of DLB [7]. In a neuropathologically confirmed study, it has been shown that in the prodromal stage of DLB, the performance in visual attention was worse than in AD [3].

To our knowledge there are no studies assessing the subfield of visuospatial and perceptual abilities in the mild cognitive impairment (MCI) stage due to DLB. Thus, the aim of this study was to determine which clinical and cognitive characteristics could help better identify MCI patients evolving to DLB from those evolving to AD, with particular emphasis on visual space and object perception deficits.

\section{Patients and Methods}

Patients were enrolled and studied at the neurological departments of the Universities of Padova and Parma (Italy). Twenty-five patients with mild cognitive deficits in the predementia stage that were eventually diagnosed with probable DLB at the follow-up visits (MCI-DLB) and 28 patients with mild cognitive deficits ultimately diagnosed as probable AD (MCI-AD) were included in the study. The charts of the patients were retrospectively evaluated, and data from clinical and neuropsychological assessments at the first visit (time of predementia stage) were evaluated by dementia experts not involved in the patients' assessments. Inclusion criteria were: absence of dementia (cutoff MMSE score $>26 / 30$ ) and of functional impairment in everyday life at the first visit, and clinical information available from the first visit to the follow-up visits until a definite clinical diagnosis of probable DLB or AD was reached according to current diagnostic criteria $[8,9]$ after at least 3 years of follow-up. Exclusion criteria were: inability to establish a definite diagnosis at the follow-up visits and cognitive decline due to comorbidities.

At the first assessment, the patients underwent full clinical history and neurological evaluation including screening for extrapyramidal signs with the Unified Parkinson's Disease Rating Scale (UPDRS) motor score [10], assessment of behavioral disorders with the Neuro- 
psychiatric Inventory Questionnaire [11], presence of fluctuations in cognition and attention using the Mayo Fluctuation Questionnaire [12] and presence of symptoms suggestive of REM sleep behavior disorder (RBD) by the Mayo Sleep Questionnaire [13].

Baseline neuropsychological evaluation was obtained for each patient by experienced neuropsychologists (N.J., F.G., S.G., C.G.). Global cognitive performance was assessed using the MMSE test. Each pentagon drawing of the MMSE test was retrospectively examined with the Qualitative Scoring MMSE Pentagon Test (QSPT) [5] by a neuropsychologist (C.B. or M.M.) not involved in the formal cognitive assessment and blind to the diagnosis. The QSPT scoring method includes the following 5 criteria of judgment: (1) number of angles; (2) distance/ intersection between the two figures; (3) closing/opening of the contour (the figure is considered closed even when two sides do not touch each other as long as the distance is $\leq 1$ $\mathrm{mm}$ ); (4) rotation of one or both pentagons (when there is no figure or the figure is not a pentagon, then the rotation is not assessable; when the rotation is less than $45^{\circ}$, the figure is not considered rotated); (5) closing-in and a total score corresponding to the sum of individual scores of each parameter, ranging from 0 to 13. For each parameter an arbitrary score was assigned, where zero indicated the worst performance. Tremor is ignored. When participants executed more than 1 copy of pentagons, the best copy was graded.

Specific cognitive domains were investigated using the following neuropsychological tests: digit cancellation and Trail Making Test A for the evaluation of visual attention; digit span forward and backward for short-term memory; prose memory (immediate and delayed recall) test for long-term memory; letter fluency test for the study of word generation and executive functioning; clock drawing test for visuoconstructional abilities and executive functions; Rey-Osterrieth Complex Figure (ROCF) test for the assessment of visuoconstructional abilities (ROCF copy) and long-term visual-spatial memory (ROCF delayed recall) [14].

The Visual Object and Space Perception (VOSP) battery was administered to all patients in order to assess visual-perceptual and visual-spatial impairments [15]. The VOSP is a neuropsychological battery composed of a screening test, 4 subtests for object perception and 4 subtests for space perception. The screening test, evaluating figure/ground perception, includes 20 squares with random patterns, on half of which a degraded ' $X$ ' is inserted. The subject is asked to judge whether the ' $\mathrm{X}$ ' is present or not (maximum score 20). For object perception, in the fragmented letters task (subtest 1 ), the subject has to recognize 20 degraded capital letters (maximum score $=20$ ); in the silhouettes task (subtest 2 ), the subject is asked to identify 30 black silhouettes presented in an unusual view (maximum score $=30$ ); in the object decision task (subtest 3), the subject has to judge which of the 4 drawings represents a real object, while the 3 other objects are nonsense drawings (maximum score $=20$ ); in the progressive silhouettes task (subtest 4), 2 series of 10 drawings progressively easier to be identified were presented (maximum score $=20$ ). For space perception, the dot counting subtest (subtest 5) is composed by 10 arrays of black dots, and the subject has to count the number of dots in each item (maximum score $=10$ ); in the position discrimination task (subtest 6), 2 squares are presented, one with a black dot printed in the center and the other one with a black dot printed out of the center: the subject has to point to the dot perceived in the center of the square (maximum sore $=20$ ); in the number location task (subtest 7), each item consists of 2 squares, one containing a random pattern of numbers and the other one containing a single black dot, corresponding to the position of one of the numbers: the subject has to point to the number that corresponds to the position of the dot (maximum score $=10$ ); in the cube analysis (subtest 8), the subject has to count how many solid bricks there are in a series of drawings, each representing a tridimensional structure of bricks (maximum score $=10$ ).

TheStudentt test for independent groups was used for the analysis of normally distributed variables, the Mann-Whitney $U$ test for ordinal variables and the $\chi^{2}$ test for categorical variables. Linear correlations between the cognitive test scores were tested using Spearman's $\rho$ 
correlation coefficient. Logistic regression model analysis was used to find the independent cognitive variables related to the diagnosis. The significance level was set at $p<0.05$.

The study received approval by the institutional review board, and all patients signed informed consent.

\section{Results}

At the MCI stage, the DLB and AD groups were matched for age (DLB: $76.5 \pm 4.9$ years, age range: $67-86$; $A D: 73.4 \pm 6.7$ years, age range: $57-83 ; p=0.06$ ), gender (male/female $=$ DLB: $14 / 11$; AD: $16 / 12 ; \mathrm{p}=0.9$ ) and education (DLB: $9.3 \pm 4.5$ years; AD: $11.4 \pm 4.7$ years; $\mathrm{p}=0.11$ ). In the DLB group, the presence of visual hallucinations was reported in $9 / 25$ patients (36\%), fluctuations of cognition and alertness in 60\% (15/25) and RBD in 60\% (15/25). In the $\mathrm{AD}$ group, 1 patient had visual hallucinations, and none manifested fluctuations or RBD.

Presence of extrapyramidal signs was the most frequent supportive feature detected in 18/25 DLB patients (72\%), although often subtle (mean UPDRS score: $4.04 \pm 5.9$ ), with 13/18 patients with UPDRS score $\leq 5$. Only 4/24 (17\%) patients with prodromal AD showed subtle extrapyramidal signs (mean UPDRS score: $0.25 \pm 0.64$ ).

Neuropsychological test results of the MCI-DLB and MCI-AD groups are reported in table 1. The mean MMSE score was $27.8 \pm 1.3$ for DLB and $27.2 \pm 1.3$ for AD ( $p=0.07)$. In the QSPT, the score for number of angles was significantly lower in the DLB patients $(p=0.01)$. An impaired number of angles (angle item score <4) was present in 44\% (11/25) of DLB patients compared to $3.7 \%(1 / 27)$ of $\mathrm{AD}(\mathrm{p}<0.001)$. No significant difference was found for the total QSPT score and its remaining subscores.

Patients of the DLB group showed greater impairment than those with AD in speed of visual attention (Trail Making Test $A, p=0.03$ ), working memory (digit span backward, $p=0.001$ ) and executive functions (verbal fluency, $p=0.002$ ). AD patients, instead, showed greater impairment in verbal episodic memory, with lower scores in prose memory delayed recall $(p=0.02)$.

As for visuospatial abilities investigated with the VOSP battery, patients with MCI-DLB presented with significantly more severe visuoconstructional deficits (cube analysis subtest) and visual-attentional deficits (number location subtest) than AD patients (table 1). Deficits in the visuoperceptual domain were detected in MCI-DLB as well as in MCI-AD, particularly in the fragmented letters, silhouettes and object decision items, and therefore did not appear to be disease specific, although more DLB patients than AD patients scored below the cutoff value of normality (i.e. silhouette item <cutoff value = DLB: 72\%, AD: 43\%) (fig. 1).

The QSPT score for number of angles correlated positively with the Trail Making Test A test score $(r=-0.34, p=0.01)$ and cube analysis of $\operatorname{VOSP}(r=0.28, p=0.04)$, and negatively with the prose memory immediate recall test score $(r=-0.36, p=0.008)$ in the whole sample. The QSPT scores for number of angles restricted to the DLB group correlated negatively only with the prose memory immediate recall $(\mathrm{p}=0.01)$.

In the multivariate analysis using a logistic regression model, we considered only the test scores that were significantly different between DLB and AD ( $\mathrm{p} \leq 0.01)$ : number of pentagon angles, digit backward, verbal fluency and cube analysis of the VOSP battery. The results of this analysis showed that only the score for number of pentagon angles and the digit backward test score remained significantly correlated with the diagnosis. Using both the score for number of angles and the digit backward tests score, the specificity (88\%) and sensitivity $(54 \%)$ of the differential diagnosis did not change significantly with respect to using only the number of angles (specificity $96 \%$, sensitivity $42 \%$ ). The positive predictive value was $81 \%$ using both variables and $91 \%$ using only the score for number of angles, while the negative predictive value was $67 \%$ and $63 \%$, respectively. 
Cagnin et al.: Clinical and Cognitive Phenotype of Mild Cognitive Impairment Evolving to Dementia with Lewy Bodies

Table 1. Cognitive tests score differences between prodromal DLB and AD patients

\begin{tabular}{|c|c|c|c|}
\hline Cognitive tests & $\begin{array}{l}\text { Prodromal DLB } \\
(\mathrm{n}=25)\end{array}$ & $\begin{array}{l}\text { Prodromal AD } \\
(n=28)\end{array}$ & $\mathrm{p}$ \\
\hline MMSE & $27.8 \pm 1.3$ & $27.2 \pm 1.3$ & 0.07 \\
\hline \multicolumn{4}{|l|}{ QSPT } \\
\hline Total score & $10.8 \pm 2.1$ & $11.6 \pm 1.2$ & 0.1 \\
\hline Number of angles & $3.28 \pm 1.1$ & $3.96 \pm 0.2$ & 0.01 \\
\hline Number of angles $<4, \%$ & 44 & 3.7 & $<0.001$ \\
\hline Digit cancellation & $41.8 \pm 8.2$ & $43.7 \pm 9.1$ & 0.56 \\
\hline Trail Making Test A, s & $99 \pm 70.8$ & $66.4 \pm 28$ & 0.03 \\
\hline \multicolumn{4}{|l|}{ Digit span } \\
\hline Forward & $4.7 \pm 1$ & $5.1 \pm 0.8$ & 0.1 \\
\hline Backward & $3.0 \pm 1.2$ & $3.9 \pm 0.6$ & 0.001 \\
\hline \multicolumn{4}{|l|}{ Prose memory } \\
\hline Immediate recall & $10.7 \pm 4.6$ & $8.2 \pm 5.9$ & 0.08 \\
\hline Delayed recall & $11.4 \pm 5.8$ & $7.7 \pm 5.4$ & 0.02 \\
\hline Verbal fluency & $22.3 \pm 10.1$ & $30.5 \pm 9$ & 0.002 \\
\hline \multicolumn{4}{|l|}{ ROCF } \\
\hline Copy & $27.1 \pm 4.9$ & $31.1 \pm 7.2$ & 0.05 \\
\hline Delayed recall & $8.9 \pm 4.5$ & $8.6 \pm 5.4$ & 1 \\
\hline \multicolumn{4}{|l|}{ VOSP } \\
\hline \multicolumn{4}{|l|}{ Visual perception } \\
\hline Fragmented letters (cutoff $\geq 16$ ) & $16.7 \pm 4.2$ & $17.3 \pm 3.7$ & 0.51 \\
\hline Silhouettes (cutoff $\geq 15$ ) & $13.9 \pm 7.2$ & $15.2 \pm 5.1$ & 0.31 \\
\hline Object decision (cutoff $\geq 14$ ) & $12.6 \pm 3.1$ & $14.6 \pm 3.8$ & 0.05 \\
\hline Progressive silhouettes (cutoff $\geq 15$ ) & $11.9 \pm 2.3$ & $11.8 \pm 2.6$ & 0.96 \\
\hline \multicolumn{4}{|l|}{ Spatial perception } \\
\hline Dot counting (cutoff $\geq 8$ ) & $9.4 \pm 0.7$ & $9.6 \pm 1.3$ & 0.81 \\
\hline Position discrimination (cutoff $\geq 18$ ) & $18.5 \pm 1.8$ & $19.1 \pm 1.8$ & 0.25 \\
\hline Number location (cutoff $\geq 7$ ) & $7.6 \pm 1.8$ & $8.9 \pm 2.5$ & 0.03 \\
\hline Cube analysis (cutoff $\geq 6$ ) & $7.3 \pm 2$ & $8.8 \pm 2.2$ & $<0.001$ \\
\hline
\end{tabular}

Data are presented as mean \pm SD except where indicated otherwise. Bold $\mathrm{p}$ values indicate statistical significance.

\section{Discussion}

\section{Clinical Features}

In the MCI stage, the best clinical predictor of the developing disease (DLB vs. AD) was presence of soft extrapyramidal signs, detected in $72 \%$ of DLB patients, followed by presence of RBD $(60 \%)$ and fluctuations (60\%). These findings confirmed that some core features of DLB are already present before an overt dementia syndrome is manifested [8]. Moreover, $52 \%$ of MCI-DLB patients presented at the first assessment with 2 core features (fluctuation and extrapyramidal involvement). However, these features may be underestimated since they could be very mild (very subtle motor involvement) or not sufficiently enquired (RBD and fluctuations). On the other side, only 4 patients with AD had 1 core feature of DLB. These data are in line with those reported in a neuropathology case series by Jicha et al. [3], in which 8 of 9 patients with MCI developing brain pathological changes supportive of DLB had at least 1 symptom among the core features of DLB versus none of the MCI subjects developing later AD.

The study by Molano et al. [2] underscores the importance to investigate symptoms and signs beyond cognition in the prodromal DLB stages and suggests that RBD may be the most 
Fig. 1. Mean percentage of correct scores for the VOSP subtests in MCI-DLB (light gray columns) and AD (dark gray columns).

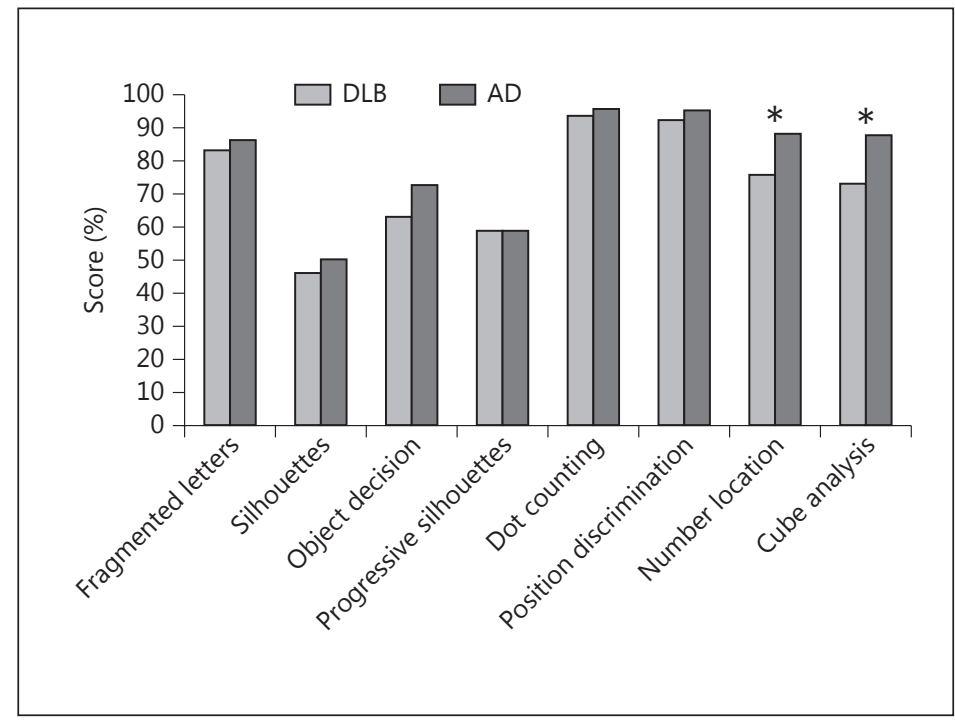

sensitive predictor of developing DLB. We confirm this statement, although in our study, the most frequent predictor of DLB was the presence of mild parkinsonism. Postuma et al. [16] studied the development of parkinsonism in a cohort of patients with idiopathic RBD and demonstrated that motor changes, assessed with the UPDRS scale, were present for 6 years before the diagnosis of DLB, although with slow progression (UPDRS increase: 2 points per year). They concluded that extrapyramidal signs in prodromal DLB have a more indolent course than in Parkinson's disease and proposed that this may be, at least in part, due to the involvement of extranigral structures. In our study, extrapyramidal signs were subtle, mostly with a UPDRS motor score $\leq 5$, suggesting the need to have a trained specialist to be intercepted. It would be interesting to assess whether mild extrapyramidal signs detectable in $\mathrm{AD}$ patients, such as in the 4 subjects of this study, are due to the comorbid presence of $\alpha$-synuclein pathology in the substantia nigra or whether they are caused by alterations of extranigral structures. In a recent retrospective cohort study of 531 patients with neuropathological verification, $\mathrm{AD}$ with Lewy bodies had poorer motor performance and more behavioral problems than AD without Lewy bodies [17].

Although the main limitation of this study is the lack of autopsy confirmation, we are confident that the clinical diagnosis of DLB would have mirrored the neuropathological changes, since the likelihood of occurrence of the clinical syndrome of DLB has been found to be positively related to the extension of cortical Lewy bodies and negatively to neuritic pathology in $\mathrm{AD}[18]$.

\section{Cognitive Features}

Prodromal AD showed a disproportionate involvement in long-term episodic memory, while prodromal DLB manifested a poorer verbal working memory (digit span backward) and a prominent difficulty in the speed and ease of verbal production (verbal fluency), as a consequence of more frontal/dysexecutive dysfunction. Our study supports the hypothesis that the visuoconstructional and object/space perception deficits typical of overt DLB are already present in the prodromal stage of the disease. However, visuoconstructional and visual motor tracking deficits were more severe in early DLB patients than in AD, while visualperceptual abilities investigated by the fragmented letters, silhouettes, object decision and progressive silhouettes items of the VOSP battery were impaired in both prodromal DLB and 
Cagnin et al.: Clinical and Cognitive Phenotype of Mild Cognitive Impairment Evolving to Dementia with Lewy Bodies

AD. We were also able to demonstrate in prodromal DLB a specific impairment in number location and cube analysis, which rely on the 'dorsal visual stream', similarly to what Calderon et al. [19] found in clinically manifested DLB. In other words, the ventral visual pathway ('what') seems to be involved in the early stages in both DLB and AD, while functional impairment of the dorsal pathway ('where') is more specific of DLB.

Presence of visual-spatial/visuoconstructional deficits has been considered very common in autopsy-confirmed DLB patients at their first assessment [20]. The authors of this study proposed that the best model to predict DLB is the presence of visual hallucinations (positive predictive value: $83 \%$ ), while lack of visual-spatial deficit represented a highly negative predictor of DLB (negative predictive value: 83\%). However, in this study the patients were demented (mean MMSE score $24 \pm 4.2$ ) and not in their prodromal stage. Our data referred to a disease stage antecedent to that described by Tiraboschi et al. [20] and strengthened the previous finding by Ferman et al. [1] which longitudinally followed a cohort of MCI-DLB patients describing a pattern of attentional and visuospatial involvement since the prodromal stage of the disease. Although the retrospective nature of our study may be a limitation, it has the advantage to investigate the full spectrum of visual-spatial perception and attention by applying a specific cognitive battery. As such, we were able to define that the visuoconstructional and spatial abilities are most specifically impaired in DLB, while object perception deficits, although very sensitive, are not specific cognitive signatures among DLB and AD in the prodromal stage.

The pentagon copying of the MMSE was first investigated in neuropathologically proven DLB and AD patients [4]. In the study by Ala et al. [4], 2 of 17 DLB patients and 17 of 26 AD patients drew the pentagons regularly (10 angles, of which 2 intersecting), reaching an $88 \%$ sensitivity and a $58 \%$ specificity in discriminating these two conditions. However, the data referred to a group of patients in the full spectrum of disease severity, while in the earlier stages specificity could increase at the expense of sensitivity.

In an autopsy-proven study of pure AD $(n=66)$ and DLB $(n=9)$ patients, as opposed to the combination of AD and DLB $(n=57)$, it was demonstrated that pure AD patients performed worse in the verbal memory domain, while pure DLB patients presented spared verbal memory and a more altered visuospatial domain [21]. The AD/DLB comorbidity influences the cognitive pattern by further impairing only the visuospatial domain. Therefore, co-occurrence of DLB and $A D$ pathology did not influence the speed of disease progression but only the type of cognitive impairment. We acknowledge that the main limitation of our study is the lack of neuropathological confirmation and the impossibility to confirm the above-mentioned data.

We found that prodromal DLB patients with reduced number of angles of the pentagon copy task, an index of altered visuoconstructional ability, have better verbal memory immediate recall scores than DLB patients with preserved number of angles. We hypothesized that this subgroup of DLB patients may represent the pure form of DLB. This suggestion is supported by the finding of Yoshizawa et al. [22] that pure DLB patients have worse visuoconstructional deficits with respect to patients with $\mathrm{AD}$ and also mixed AD/DLB since the early stage of the disease. The confirmation of clinical diagnosis by brain neuropathology will validate this hypothesis.

\section{Disclosure Statement}

There are no conflicts of interest. 
Cagnin et al.: Clinical and Cognitive Phenotype of Mild Cognitive Impairment Evolving to Dementia with Lewy Bodies

\section{References}

1 Ferman TJ, Smith GE, Kantarci K, Boeve BF, Pankratz VS, Dickson DW, Graff-Radford NR, Wszolek Z, Van Gerpen J, Uitti R, Pedraza O, Murray ME, Aakre J, Parisi J, Knopman DS, Petersen RC: Nonamnestic mild cognitive impairment progresses to dementia with Lewy bodies. Neurology 2013;81:2032-2038.

$>2$ Molano J, Boeve B, Ferman T, Smith G, Parisi J, Dickson D, Knopman D, Graff-Radford N, Geda Y, Lucas J, Kantarci K, Shiung M, Jack C, Silber M, Pankratz VS, Petersen R: Mild cognitive impairment associated with limbic and neocortical Lewy body disease: a clinicopathological study. Brain 2010;133:540-556.

-3 Jicha GA, Schmitt FA, Abner E, Nelson PT, Cooper GE, Smith CD, Markesbery WR: Prodromal clinical manifestations of neuropathologically confirmed Lewy body disease. Neurobiol Aging 2010;31:1805-1813.

4 Ala TA, Hughes LF, Kyrouac GA, Ghobrial MW, Elble RJ: Pentagon copying is more impaired in dementia with Lewy bodies than in Alzheimer's disease. J Neurol Neurosurg Psychiatry 2001;70:483-488.

5 Caffarra P, Gardini S, Dieci F, Copelli S, Maset L, Concari L, Farina E, Grossi E: The qualitative scoring MMSE pentagon test (QSPT): a new method for differentiating dementia with Lewy body from Alzheimer's disease. Behav Neurol 2013;27:213-220.

6 Caffarra P, Vezzadini G, Dieci F, Zonato F, Venneri A: Rey-Osterrieth complex figure: normative values in an Italian population sample. Neurol Sci 2002;22:443-447.

7 Mitolo M, Salmon DP, Gardini S, Galasko D, Caffarra P: The new Qualitative Scoring MMSE Pentagon Test (QSPT) as a valid screening tool between autopsy-confirmed dementia with Lewy bodies and Alzheimer's disease. J Alzheimers Dis 2014;39:823-832.

8 Cagnin A, Bussè C, Jelcic N, Gnoato F, Mitolo M, Caffarra P: High specificity of MMSE pentagon scoring for diagnosis of prodromal dementia with Lewy bodies. Parkinsonism Relat Disord 2015;21:303-305.

-9 McKeith IG, Dickson DW, Lowe J, Emre M, O’Brien JT, Feldman H, Cummings J, Duda JE, Lippa C, Perry EK, Aarsland D, Arai H, Ballard CG, Boeve B, Burn DJ, Costa D, Del Ser T, Dubois B, Galasko D, Gauthier S, Goetz CG, Gomez-Tortosa E, Halliday G, Hansen LA, Hardy J, Iwatsubo T, Kalaria RN, Kaufer D, Kenny RA, Korczyn A, Kosaka K, Lee VM, Lees A, Litvan I, Londos E, Lopez OL, Minoshima S, Mizuno Y, Molina JA, Mukaetova-Ladinska EB, Pasquier F, Perry RH, Schulz JB, Trojanowski JQ, Yamada M: Diagnosis and management of dementia with Lewy bodies: third report of the DLB Consortium. Neurology 2005;65:1863-1872.

-10 McKhann GM, Knopman DS, Chertkow H, Hyman BT, Jack CRJ, Kavas CH, Klunk WE, Koroshetz WJ, Manly JJ, Mayeux R, Mohs RC, Morris JC, Rossor MN, Scheltens P, Carrillo MC, Thies B, Weintraub S, Phelps CH: The diagnosis of dementia due to Alzheimer's disease: recommendations from the National Institute on AgingAlzheimer's Association workgroups on diagnostic guidelines for Alzheimer's disease. Alzheimers Dement 2011;7:263-269.

11 Fahn S, Elton RL; Members of the Unified Parkinson's Disease Rating Scale Development Committee: Unified Parkinson's disease rating scale; in Fahn S, Marsden CD, Calne DB, et al. (eds): Recent Developments in Parkinson's Disease. New York, McMillan Healthcare Information, 1987, pp 153-164.

$\checkmark 12$ Cummings JL: The Neuropsychiatric Inventory: assessing psychopathology in dementia patients. Neurology 1997;48:S10-S16.

13 Ferman TJ, Smith GE, Boeve BF, Ivnik RJ, Petersen RC, Knopman D, Graff-Radford N, Parisi J, Dickson DW: DLB fluctuations: specific features that reliably differentiate DLB from AD and normal aging. Neurology 2004;62: 181-187.

14 Boeve BF, Molano JR, Ferman TJ, Smith GE, Lin SC, Bieniek K, Haidar W, Tippmann-Peikert M, Knopman DS, Graff-Radford NR, Lucas JA, Petersen RC, Silber MH: Validation of the Mayo Sleep Questionnaire to screen for REM sleep behaviour disorder in an aging and dementia cohort. Sleep Med 2011;12:445e53.

15 Warrington EK, James M: Visual Object and Space Perception Battery. Bury St Edmunds, Thames Valley Test Company, 1991.

16 Postuma RB, Lang AE, Gagnon JF, Pelletier A, Montplaisir JY: How does parkinsonism start? Prodromal parkinsonism motor changes in idiopathic REM sleep behaviour disorder. Brain 2012;135:1860-1870.

17 Chung EJ, Babulal GM, Monsell SE, Cairns NJ, Roe CM, Morris JC: Clinical features of Alzheimer disease with and without Lewy bodies. JAMA Neurol 2015;72:789-796.

18 Tiraboschi P, Attems J, Thomas A, Brown A, Jaros E, Lett DJ, Ossola M, Perry RH, Ramsay L, Walker L, McKeith IG: Clinicians' ability to diagnose dementia with Lewy bodies is not affected by $\beta$-amyloid load. Neurology 2015;84:496-499.

19 Calderon J, Perry RJ, Erzinclioglu SW, Berrios GE, Dening TR, Hodges JR: Perception, attention, and working memory are disproportionately impaired in dementia with Lewy bodies compared with Alzheimer's disease. Neurol Neurosurg Psychiatry 2001;70:157-164.

20 Tiraboschi P, Salmon DP, Hansen LA, Hofstetter RC, Thal LJ, Corey-Bloom J: What best differentiates Lewy body from Alzheimer's disease in early-stage dementia? Brain 2006;129:729-735.

21 Johnson DK, Morris JC, Galvin JE: Verbal and visuospatial deficits in dementia with Lewy bodies. Neurology 2005;65:1232-1238.

22 Yoshizawa H, Vonsattel JPG, Honig LS: Early neuropsychological discriminants for Lewy body disease: an autopsy series. J Neurol Neurosurg Psychiatry 2013;84:1326-1330. 